\title{
Seasonality of Post-capture Longevity in a Medically-Important Mosquito (Culex pipiens)
}

\begin{abstract}
Nikos T. Papadopoulos ${ }^{1 * t}$, James R. Carey ${ }^{2,3+}$, Charalampos S. Ioannou ${ }^{1}$, Hao Ji ${ }^{4}$, Hans-Georg Müller ${ }^{4}$, Jane-Ling Wang ${ }^{4}$, Shirley Luckhart ${ }^{2,5}$ and Edwin E. Lewis ${ }^{2}$

${ }^{1}$ Department of Agriculture, Crop Production and Rural Environment, University of Thessaly, Magnisias, Greece, ${ }^{2}$ Department of Entomology and Nematology, University of California, Davis, Davis, CA, USA, ${ }^{3}$ Center for the Economics and Demography of Aging, University of California, Berkeley, Berkeley, CA, USA, ${ }^{4}$ Department of Statistics, University of California, Davis, Davis, CA, USA, ${ }^{5}$ Department of Medical Microbiology and Immunology, University of California, Davis, Davis, CA, USA
\end{abstract}

The epidemiological importance of the age structure and longevity potential of wild populations of mosquito disease vectors has been known for over 60 years. However, no routine method currently exists that provides reliable insights into the population age dynamics of this medically important group of insects. In this paper we use a technique originally developed for studying wild fruit fly populations to study the post-capture longevity dynamics in populations of the West Nile virus mosquito Culex pipiens in Greece. This approach, referred to as the captive cohort method, analyzes and interprets the longevity trends in wild-caught $C x$. pipiens to infer demographic changes in their field population. Approximately 10 adult females were captured each day from June through November, housed in individual cages in the laboratory, and their remaining longevity recorded. Strong differences were observed in the mean, variation, and extremes of post-capture longevity. Early season (June-July) mosquitoes lived the shortest and late season the longest with a clear transition period in September. The mean levels of post-capture longevity were quite high at over 2 months in early season to over 85 days in late season when the vast majority of adults were nulliparous and likely preparing for hibernation. Implications for both basic and epidemiological research on the biodemography of aging in the wild are discussed.

Keywords: biodemography, captive cohort, age structure, lifespan, epidemiology, aging in wild, vector biology

\section{INTRODUCTION}

A staggering $10 \%$ of the world's population (i.e., 700 million) acquires one or more mosquitoborne diseases every year including malaria, yellow fever, dengue, filariasis, and West Nile virus (Anon, 2014; Caraballo and King, 2014) and, more recently, Zika virus (Vogel, 2016). One of the most challenging problems in the development of intervention programs designed to control or eradicate the mosquito vectors of these diseases is developing, protocoling and routinizing methods for estimating the longevity potential and age structure of their populations (Cook et al., 2006, 2008; Styer et al., 2007).

The epidemiological importance of vector age has been known for nearly 60 years (MacDonald, 1957). First, because the longer female mosquitoes survive the greater is their ability to transmit the disease-causing pathogen (James et al., 2013). Older females contribute disproportionately to 
transmission because of the cumulative time required for them to mature, acquire a blood meal from an infected individual, incubate the pathogen, and transmit the pathogen via subsequent blood meals. Second, adults of many mosquito species have evolved lifespan-extending adaptations for surviving shortterm, acyclic exigencies as well as changes in the environment such as quiescence, hibernation, and migration (Tauber et al., 1986). Thus, the ability to extend potential lifespan by employing behavioral strategies (e.g., reduced host seeking) and physiological mechanisms (e.g., increased fat reserves) also has profound epidemiological implications-i.e., extended longevity allows disease-carrying adults to overwinter and serve as a disease reservoir the following spring.

Although past field studies using reproductive age grading were considered the gold standard for determining the reproductive age of field-caught females (Reisen et al., 1981), this method has fallen out of favor in recent years, because of artifacts caused by oocyte degeneration in laboratory studies (Fox and Brust, 1994) and the presence of autogeny in some Culex spp. (Nelson and Milby, 1982). Other methods, such as the use of infra-red spectroscopy (Mayagaya et al., 2009), cuticular hydrocarbons (Desena et al., 1999), pteridine concentrations (Lardeux et al., 2000), and gene profiling (Joy et al., 2012) tend to be expensive, labor intensive, use time-grouped data, and do not provide epidemiologically relevant information similar to the number of gonotrophic cycles completed. Alternatively, cohorts of adults of known age can be marked and released and then recaptured over time to measure survivorship by the decline in the recapture rate as a function of time (Reisen et al., 1980), but mortality here is confounded by losses due to emigration and removal sampling.

Because of the wide-spread recognition of the importance of longevity potential and population aging in mosquito vector biology, and the limitations of the current age estimation methods, we designed a study based on an approach developed for use in studying aging in fruit fly populations. Referred to as the captive cohort method, the technique is based on the concept that the distribution of remaining lifespans for wildcaught individuals can be used to infer the age structure dynamics of wild populations (Müller et al., 2004; Carey et al., 2012a,b). The post-capture lifespans of wild-captured individuals has direct relevance to the study of mosquito populations since remaining longevity is conditional on (and thus an expression of) their physiological and chronological ages (Klowden and Lea, 1980).

We have two general goals in this current paper. First, to describe the results of a field study in Greece on adult female West Nile virus mosquitoes, Culex pipiens (Pervanidou et al., 2014) in which the post-capture lifespans of over 1500 individuals were monitored over the field season from June through December. Second, to consider these results in the complementary contexts of both basic and applied science, the former involving demography and the biology of aging, and the latter involving medical entomology and epidemiology of mosquito-borne diseases. Earlier studies regarding fruit flies (Müller et al., 2004; Carey et al., 2012a,b) involved the use of post-capture longevity data to estimate age structure. However, in the current case we focus on the use of these same types of data on mosquitoes to infer changes in the underlying frailty structure and longevity potential but, for reasons which will become evident in the results, do not attempt to estimate age structure, per se.

\section{MATERIAL AND METHODS}

\section{Empirical}

Adult mosquitoes were sampled nightly from June through November 2013 in a semi-urban area of Nea Agchialos Magnisias $\left(39.265676^{\circ} \mathrm{N}, 22.814840^{\circ} \mathrm{E}\right)$, a town in the coastal area of the Magnesia prefecture (15 Km south of Volos), Central Greece. The sampling site was 0.4 ha garden (with several ornamental plants and fruit trees) close to a wetland bearing many mosquito breeding sites. Following unsuccessful preliminary trials to (a) recover Culex spp. mosquitos from CDC mini light traps, and (b) aspirate them from light sources we followed a third option where (c) mosquitos were allowed to enter during night into a humid empty shelter $\left(\approx 20 \mathrm{~m}^{2}\right)$ and aspirated from resting sites early next morning. The sampling area was isolated from human houses and animal shelters although sheep herds were often grazing nearby. Following this mode of sampling, we recovered large numbers of both males and females from which a sample of live females were collected for our studies.

A random daily sample of 10 Culex spp. females were individually placed into $400 \mathrm{ml}$ transparent plastic cages with a side opening $\left(35 \mathrm{~cm}^{2}\right)$ covered with muslin, provided with $10 \%$ sugar solution, and transferred to a laboratory insectary $\left(25^{\circ} \mathrm{C}, 65 \% \mathrm{RH}, 14: 10 \mathrm{~L}: \mathrm{D}\right)$ where their remaining lifespans were monitored daily. Upon placement into the plastic cages, females were visually inspected and classified as gravid (enlarged abdomens), recently blood-fed (dilated red abdomens) or nongravid (flat abdomens). To ensure stable food quality during captivity, sugar solution was renewed every week in each cage. Using appropriately modified dichotomous keys all individuals were identified to species level at death. Nearly all (98.5\%) adult mosquitoes captured were $C x$. pipiens. Morphologically similar to $C x$. pipiens species, such as $C x$. quinquefasciatus, $C x$. austalicus, and $C x$. globocoxitus are not present in southern Europe; therefore, there are no misidentification issues at the species level (Frajollahi et al., 2011). On the other hand, Cx. pipiens has two distinct biotypes "pipiens" and "molestus," which are morphologically identical but differ in behavior and physiology and cannot be separated without molecular tools. Cx. pipiens molestus is stenogamous (copulation can take place in confined spaces), autogenus (ability to develop eggs without a blood meal), homodynamic (continuous breading throughout the year), and mammophilic (feeds readily on mammals, including humans). On the other hand, Cx. pipiens pipiens is eurygamous (copulation takes place in open spaces), anautogenous (blood feeding is necessary for eggs development), heterodynamic (forgoes winter diapause), and rather ornithophilic (prefers to feed on birds; Vinogradova, 2000). The two biotypes co-occur in Grecce and hybridization between them has been reported (Gomes et al., 2013). Temperature and relative humidity data were collected throughout the experimental period from the sampling shelter using a $\mathrm{HOBO}^{\circledR}$ data logger. External meteorological data were 
obtained from a station located $4 \mathrm{~km}$ from the New Angchialos airport.

\section{Post-Capture Longevity Assay as Estimate for Wild Biological Age Index}

It is well-known that, like all insects, the longevity of adult mosquitoes is determined by their thermal environment as well as by a range of additional factors such as larval conditions and adult nutritional (e.g., blood feeding) ecology. Thus, a more meaningful and useful age concept applied to wild populations is biological age rather than chronological age. Inasmuch as remaining longevity is widely considered to be a manifestation of biological age more so than chronological age (see Levine, 2012 and references therein), we use remaining longevity as a proxy for a mosquito's biological age at time of capture. For example, a group of individuals that lived (hypothetically) 30 days under the relatively cool spring conditions in the wild pre-capture would, under our assumptions, be considered to be the same biological age as a group of individuals that lived 10 days under much hotter conditions in mid-summer pre-capture if each experienced the same remaining life expectancy post-capture. Similar concepts would apply to two populations that are subject to different larval conditions and/or blood feeding patterns but exhibited similar post-capture longevity patterns. Therefore, we interpret differences in post-capture longevity patterns as due to differences in population biological age structure without regard to the multiplicity of coalescing environmental, ecological and behavioral longevity determinants underlying the differences.

\section{Data Analysis and Statistical Methods}

Since all animals were followed until death, no censoring effect is present in the data. Cubic splines were used to smooth the weekly mean remaining lifespans. The smoothed curve shows an increasing trend from summer to late autumn. In order to analyze the calendar time effects on distribution of remaining lifespan, we examined the distributions of weekly and monthly aggregated captive lifetimes and used the Kruskal-Wallis test, which is a surrogate for one-way ANOVA with relaxed distribution assumption, to bi-weekly, monthly and seasonally aggregated data. For analyzing survival, we used the Kaplan Meier estimator for daily aggregated data.

\section{RESULTS}

\section{Raw Post-Capture Longevity Data and Daily Means}

The post-capture lifespans for a total of 1504 Cx. pipiens females were monitored over the 170-day period between June 8 and November 25, 2013. Mean longevity for these wild-caught individuals was 66.3 (SD 25.45) days with a range of 1 to 139 days (4.5 months). The percentage that survived $2-3,3-$ 4 , and $>4$ months was $60.0,17.5$, and $2.5 \%$, respectively, with only $2.5 \%$ surviving 2 weeks or less. Post-capture longevity was distinctly as evident in the raw data (Figure 1A) and daily means (Figure 1B). Whereas post-capture longevity was lowest in early season averaging between 50 and 60 days, it was highest in late season when it averaged 75 to 85 days. The majority of the

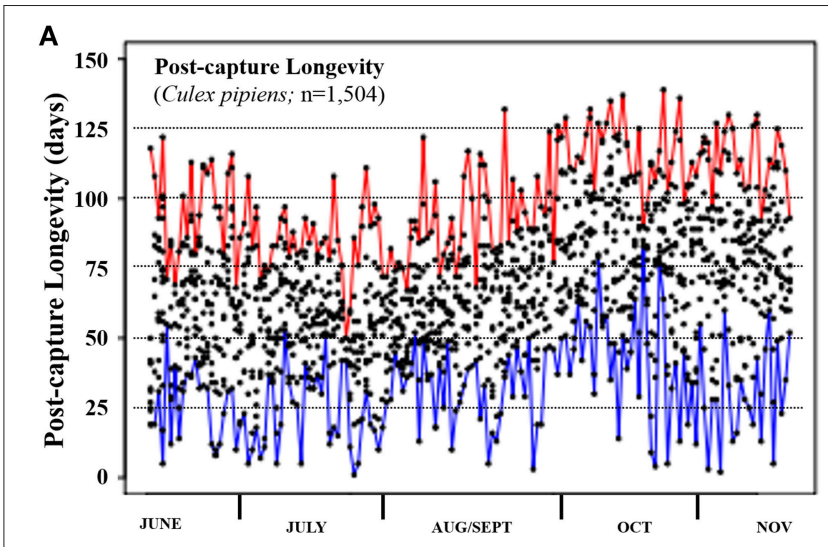

B

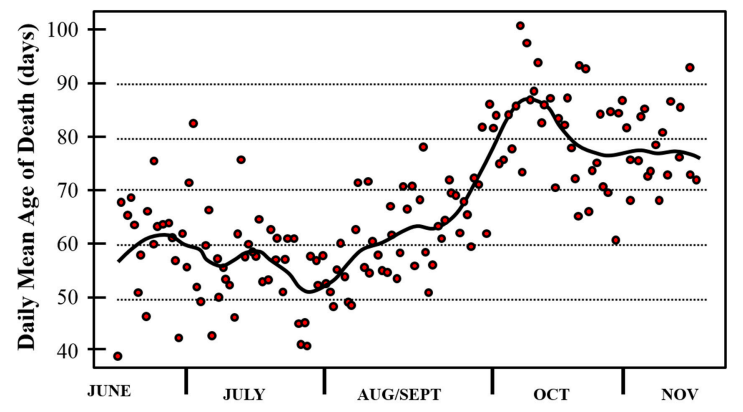

FIGURE 1 | Post-capture longevity for the mosquito Cx. pipiens captured in Volos, Greece, from June through November 2013. (A) Raw data; red and blue lines describe the maximum and minimum remaining lifetime for each day. (B) Each point depicts average post-capture longevity for the $\approx 10$ individuals sampled each day.

shorter-lived adults were captured in the summer and virtually all of the longest-lived adults (i.e., lived $>125$ days) captured in the fall. The daily means of post-capture longevity (Figure 1B) varied by over 40 days ranging from less than 50 days in summer months to over 90 days in mid- and late-fall.

\section{Boxplots}

We visualized the data aggregated at fine-grained (i.e., weekly), course-grained (monthly), and dichotomized (seasonally) intervals to provide different perspectives and insights into the post-capture data. The post-capture lifespans differ significantly between months and weeks of capture $(p<0.00001$ for both Kruskal-Wallis tests).

Based on the totals for each of 24 weekly captures ( $n$ $\approx 60$ adults/week), the weekly boxplots (Figure $2 \mathrm{~A}$ ) reveal that, although mean post-capture longevity remained relatively steady at around 55-60 days for the first 2 months (8 weeks) of the season, the interquartile range (IQR; 25-75\%) decreased significantly $(p<0.0001$ comparing IQR of first and second 4 weeks). The IQR in the first week was over 40 days which then shortened to around 20 days 8 weeks later. This trend was reversed in the following 8 weeks. In particular, the mean postcapture longevity increased by more than 30 days from 55 to over 85 days, while during the same period the IQR remained 

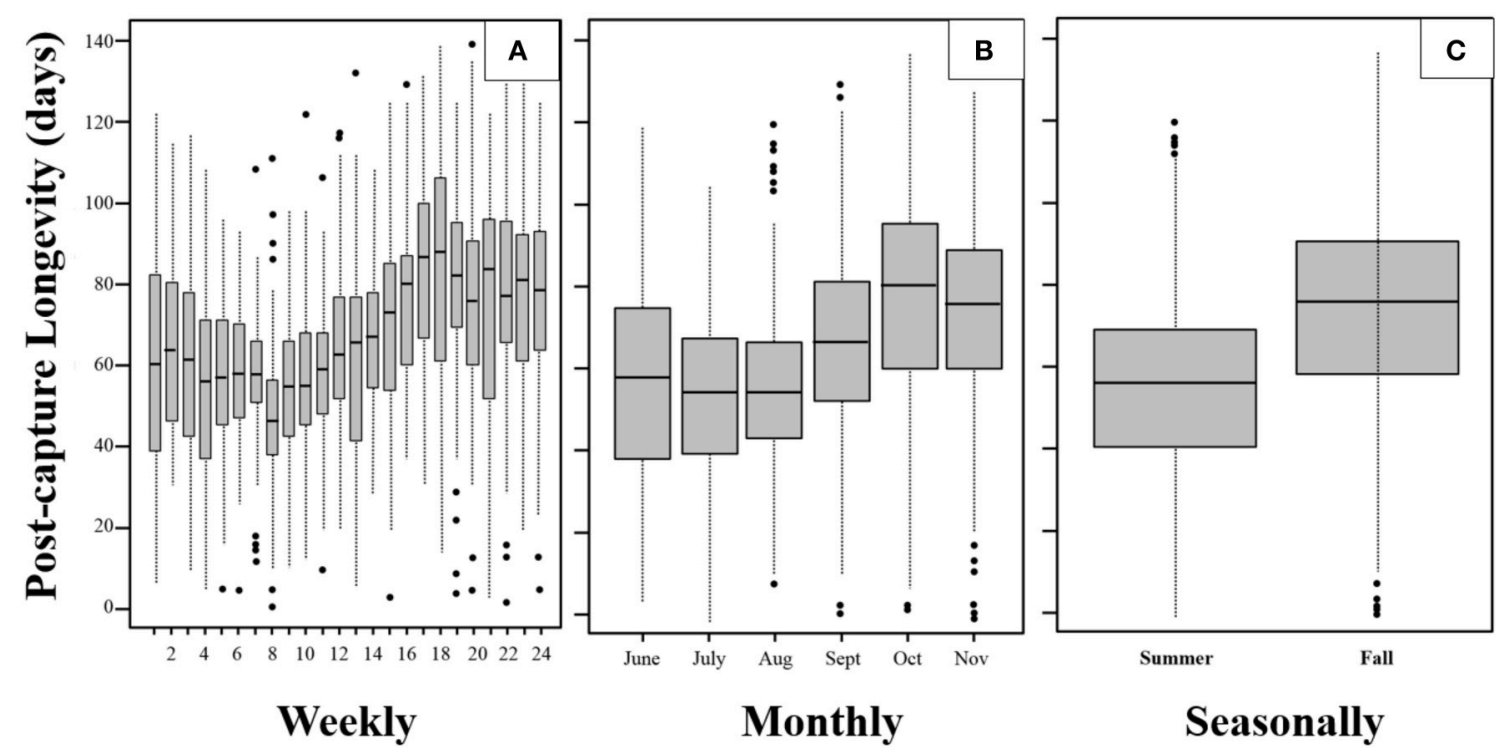

FIGURE 2 | Boxplots of weekly (A), monthly (B), and (C) seasonal post-capture longevity for the mosquito Cx. pipiens captured in Volos, Greece. Midline of each box is the median Q2, lower and upper lines are the first Q1 and third Q3 quartile. Interquartile range is IQR = Q3 - Q1 and the whiskers represent minimum or maximum lifetime if there are no outliers present. If there are outliers present (appearing as dots), then the whiskers correspond to points that are 1.5 ICR below Q1 or above Q3.

at around 20 to 25 days. In mid- to late-fall (September to November) both the mean post-capture longevity and the IQR were relatively high and constant. The boxplots in Figure 2B both reinforce and summarize the trends noted in Figure $\mathbf{2 A}$.

Using the totals for summer and fall ( $n \approx 750$ adults/period), the boxplots in Figure $2 \mathrm{C}$ show the large differences in postcapture longevity of mosquitoes captured in the summer vs. those captured in the fall. Note that the third quartile of post-capture longevity for the summer months overlaps substantially with the second quartile for the fall months. Whereas the outliers occurred in the upper post-capture ages in the summer (i.e., extremely long post capture survival the exception this period), the outliers occurred in the lower post-capture ages in the fall (i.e., extremely short post-capture survival the exception this period).

\section{Post-Capture Survival}

One of the most striking qualitative aspects of the survival curves corresponding to monthly post-capture survival of $C x$. pipiens (Figure 3) is the similarity in their shapes-i.e., all are convex with gentle downward slopes at younger post-captures ages followed by more-or-less linear declines with tails of various lengths. In no month was the shape of the survival curve either concave (i.e., sharply decreasing survival at younger post-capture ages) or "squared" (i.e., extremely high survival rates at younger and middle ages followed by sudden, steep declines at older ages). Differences in the post-capture ages at which $50 \%$ of newlycaptured $C x$. pipiens were dead were much greater with the reduction in June- and October-captured mosquitoes occurring at 61 days and 84 days, respectively-a difference of 23 days. Fewer than $1 \%$ (standard error $0.35 \%$ ) of individuals captured in June and July survived to 115 days, but $8.8 \%$ (s.e. $1.68 \%$ ) and

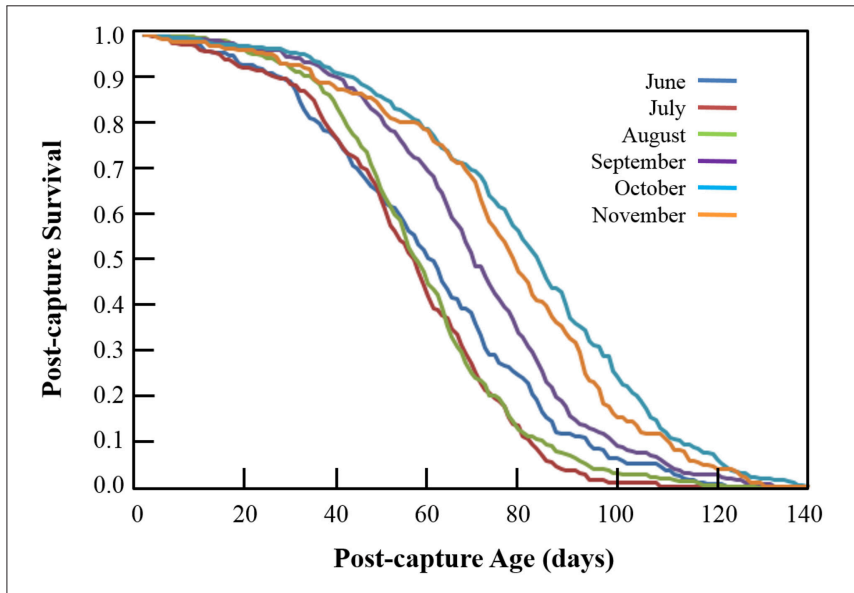

FIGURE 3 | Monthly post-capture survival of adult female Cx. pipiens from Volos, Greece in 2013. Each curve represents the fraction of individuals captured each month that survive to the corresponding post-capture age.

$5.7 \%$ (s.e. $1.61 \%$ ) of those captured in October and November, respectively, survived to this post-capture age.

\section{Reproductive Status and Post-Capture Lifespans}

The percentage of gravid females dropped from $60 \%$ in August to $47.4 \%(p=0.0063)$ and $15.1 \%(p<0.0001)$ in September and October (Table 1). Post-capture longevity was highly variable in the non-gravid cohort with females captured in October living 25 days (or 1.5 times) longer than those captured in August, and the $95 \%$ confidence interval of the mean difference is $(19.35$, 
TABLE 1 | Captive longevity (in days) of female $C x$. pipiens that were captured in August, September and October and were classified as either non-gravid or gravid based on visual observation.

\begin{tabular}{lcccc}
\hline Month of Capture & \multicolumn{4}{c}{ Average longevity (95\% Confidence intervals) } \\
\cline { 2 - 5 } & $\boldsymbol{n}$ & Non-gravid & $\boldsymbol{n}$ & Gravid \\
\hline August & 108 & $57.2(53.3-61.1)$ & 162 & $58.7(55.7-61.7)$ \\
September & 122 & $68.9(64.6-73.1)$ & 110 & $70.4(66.3-74.6)$ \\
October & 241 & $81.7(78.3-85.1)$ & 43 & $72.6(64.6-80.7)$ \\
\hline
\end{tabular}

29.65). Interestingly, longevity of gravid/non-diapausing females captured in September and October significantly increased compared to those captured in August [95\% C.I. of mean difference $(7.62,17.09)]$. The percentage of gravid females with post-capture lifespans $>80$ days increased from $12.96 \%$ in August to $20.90 \%$ in September and $31.82 \%(p<0.001)$ in October. Likewise, the percentage of gravid females surviving $>100$ days rose from $1.2 \%$ in August to $7.3 \%(p=0.023)$ and $13.95 \%$ ( $p=0.33$ between September and October) in September and October, respectively.

\section{Seasonality of Cx. pipiens Captive Lifespan and Its Chronological Reach}

Plots of the longevity of individual mosquitoes relative to both time of year and start of season are presented in Figure 4, several aspects of which merit comment. First, survival of mosquitoes captured in late fall was extremely high with nearly $20 \%$ (s.e. $2.4 \%$ ) of mosquitoes captured in October (2013) surviving to February (2014). Over 50\% (s.e. 3.46\%) of individuals captured in November survived to February and $10 \%$ (s.e. $2.1 \%$ ) survived to March. Second, most of the oldest mosquito life-days in the population were lived in the late winter and early spring of the next year. Based on results shown in this graphic as well as those presented earlier, we suggest that $C x$. pipiens populations undergoes three demographic phases: (1) Recurrent Phase. This occurs from the earliest generations in the season through midto late-summer. Females are actively seeking blood meals and lifespans are potentially long but ordinary; (2) Transition Phase. Average longevity in the population increases as females begin to shift to hibernation physiology; (3) Hibernation Phase. Longevity potential for females is extremely high as they cease blood feeding and reproduction and build up their fat reserves for overwintering.

\section{DISCUSSION}

The current study concerned with understanding aging and longevity potential in wild populations of the West Nile virus mosquito (Cx. pipiens) was motivated by two interrelated factors: (1) the widespread recognition that mosquito age and longevity potential play an extraordinarily important role in their ability to transmit pathogens (i.e., adult mosquitoes must survive long enough to blood feed at least twice with the interim period between feedings equaling or exceeding the pathogen's incubation period); and (2) the absence of routinized methods for tracking either. Thus, our goal in this paper was to introduce a method that we believe has the potential to use the post-capture longevity of wild-caught mosquitoes as a proxy for tracking changes in the longevity potential of populations.

This relationship between disease transmission and long life also holds for other species of arthropod vectors including Simulium black flies, Phlebotomine sand flies, ticks, and triatomine bugs that transmit river blindness, leishmaniasis, haemorrhagic fever, and Chagas disease, respectively. With half of the world population at risk of vector-borne diseases and a sixth of all illnesses and disabilities suffered worldwide caused by vector-borne pathogens (WHO, 2014)., it is hard to overstate the importance of developing a method for understanding the age dynamics of wild vector populations to inform policies concerned with prediction and intervention.

In the following sections we outline and discuss the basic and applied implications of both our results and the methods we used to acquire them.

\section{Demographic Inferences Longevity Potential}

One of the most remarkable discoveries of this study was the extraordinary longevity (i.e., averages of 60 to 90 days) of wildcaught $C x$. pipiens. This was true not just for a select subset of the wild-caught mosquitoes or for individuals captured in a narrow window (e.g., pulse of new-borne), but for the vast majority of those captured throughout the field season. That individuals that have already lived a fraction of their lives (in the wild) prior to capture and laboratory monitoring are capable of living an average of 50-85 days depending on time of year, is as demographically noteworthy as it is epidemiologically important. This longevity was several-fold greater than the results from a number of life table laboratory studies of Cx. pipiens (Walter and Hacker, 1974; Gomez et al., 1977; Wang, 1998; Alto et al., 2014).

\section{Pre-Hibernation Transition}

Adult female $C x$. pipiens are the overwintering stage in climatic regions with temperate climates like Greece where they use natural shelters (e.g., caves, hollows, holes) and artificial refuges (e.g., cellars, sheds, catacombs) as hibernacula (Vinogradova, 2000). In light of the large increases in longevity potential and percentages of non-gravid females in the fall months, the most logical explanation is that these changes in the population were the result of changes in adult females preparing for overwintering. These changes include biological factors such as the accumulation of fat reserves and the arrest of ovary development, as well as behavioral changes such as reduced host seeking (Vinogradova, 2000; Denlinger and Armbruster, 2014). The magnitude of potential longevity increase from around 50 or 60 days in the summer months to around 75 and 85 days in the fall (i.e., 15-30 days) sheds new and important demographic light on how this species survives the winter months-i.e., low temperature combined with an increase in potential longevity (relative to longevity potential under midseason climatic conditions) of two to 3 weeks. 


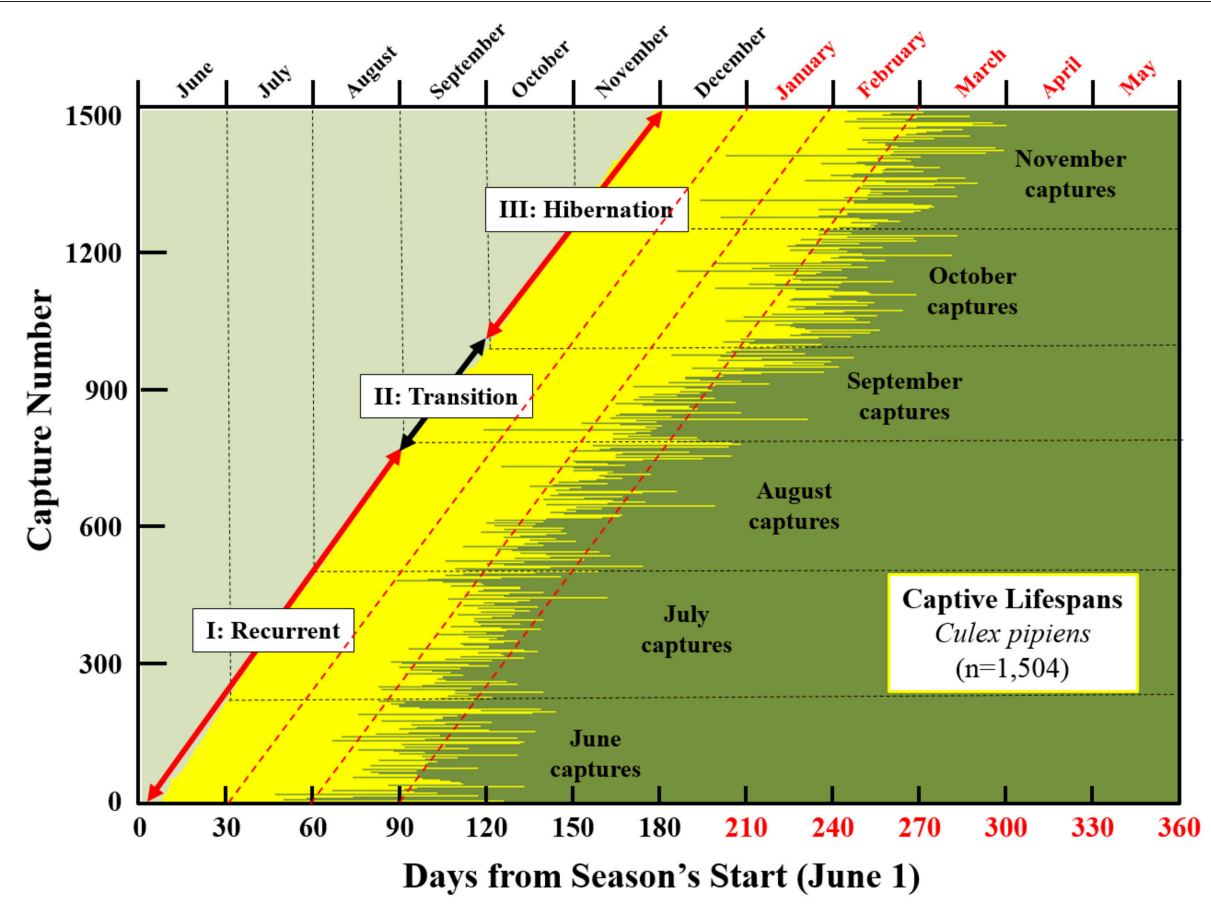

FIGURE 4 | Seasonality of longevity and its chronological reach for $\sim 1500$ Cx. pipiens females live-captured in Greece in 2013 and the proposed demographic stages of the population (recurrent, transition, and hibernation). Red fonts (top and bottom right) indicate days and months in 2014 . Captive lifespans of individual females, depicted as horizontal lines starting at date of capture and ending at date of death, progress sequentially according to sampling date from bottom diagonally to top. Time scales are both absolute (i.e., by month in top axis) and relative (days from June 1 in bottom axis) with red fonts indicating months (top) or days (bottom) in the next year (i.e., starting in January). The dashed diagonal red lines indicate 30, 60, and 90day post-capture longevities. Cross-sectional perspectives on overlapping generations can be visualized as vertical transects. For example, the mixture of ages for individuals captured in June that were still living in September. Or for individuals captured in November that survived the winter months to the following March.

\section{Age-Structure Dynamics}

Even though we did not attempt to estimate age structure, per se, in this wild $C x$. pipiens population based on post-capture longevity data, we believe the information provides important insights into at least three aspects of the dynamics of biological age including: (1) moderate changes (i.e., 10-15 days) in the mean post-capture age during the summer months suggest that there were likely changes in the $C x$. pipiens population age structure of roughly the same magnitude; (2) the increase in mean post-capture longevity during September to levels not observed in earlier months along with the simultaneous increase in IQR suggests that the number of age classes were increasing and that most or even all age classes in the wild Cx. pipiens population were beginning to transition to the hibernation mode; and (3) the extraordinarily long post-capture lifespans in October combined with both the large IQR and the observations that the vast majority of adults captured this month were nulliparous, suggests that virtually all individuals in the population were in the hibernation mode.

\section{Applications}

\section{Mosquito Vector Monitoring}

Vector research and control program surveys are generally classed into one of four categories (Anon, 2003), including preliminary (e.g., baseline data), regular or trend (e.g., information on changes in vector density), spot checks (e.g., supplement routine observations, and focal investigations (new or persistent disease transmission). For small cost, minimal effort and simple technology requirements, monitoring regional patterns in longevity potential of live-caught mosquitoes as in this study have the potential to provide insights into trends in the age structure of mosquito populations and their vectorial capacity. These include trends in population age, population pulses as inferred from the sudden appearance of long-lived mosquito subgroups, age shifts both pre-and post-intervention, and comparison of age bias in sampling (see Kouloussis et al., 2009).

\section{Research}

The approach outlined in this paper has the potential to provide new insights into research designed to better understand the ecology and demography of vector populations. For example, studies of the Sahelian malaria mosquito populations by Dao and his colleagues (Dao et al., 2014) were based largely on the timing of species-specific captures to understand what they termed the "dry season paradox"-that dormancy shapes vector composition in the Sahel region of North Africa. These researchers were interested in how vectors survive the dry season when there is little water in which mosquitoes can lay their eggs. They concluded that Anopheles gambiae and Anopheles 
arabiensis undergo long distance migration, but that Anopheles coluzzii persists locally in a form of diapause. That is, two former species recolonize the area from refugia hundreds of kilometers distant, while the latter re-emerges locally. This is an example of important research in which supporting data used to test their hypotheses could have been enhanced with information on postcapture longevity from each of the species throughout the 3-year sampling period.

\section{Mosquito Life Shortening Strategies}

Because pathogens transmitted by mosquitoes share the common property that they must undergo an incubation period in the insect vector before they can be transmitted, targeting mosquito age with the inherited bacterial symbionts in the genus Wolbachia may be a viable strategy to reduce the transmission of pathogens such as dengue viruses (Brownstein et al., 2003; McMeniman et al., 2009). Methods outlined in this paper could be used to measure changes in age structure in field populations for trial programs that use Wolbachia as a life-shortening strategy in malaria control. Cook et al. (2008) noted that one of the major challenges associated with the development of control strategies that reduce vector longevity is the need to identify methods that can accurately monitor changes in the age structure of mosquito populations.

\section{Epidemiology}

Insights into the demography of wild mosquito populations are important in two interrelated contexts: pathogen transmission and pathogen persistence. Information on the age structure of Anopheles mosquito populations is essential to assess the impact of environmental and climate changes and vector control measures on malaria parasite transmission (Mia et al., 2012; Wang et al., 2013). In the context of West Nile virus, Nasci and co-workers (Nasci et al., 2001) suggested that virusinfected, hibernating $C x$. pipiens likely play an important role in persistence of the virus in an area. Denlinger and Armbruster (2014) note that hibernating mosquitoes are important for virus transmission because they serve as reservoirs to re-establish transmission the following season.

\section{CONCLUSIONS}

The main strength of this paper is that it describes the results of the first study that we are aware of that measures systematically the remaining longevity of wild-caught mosquitoes and, in turn, uses these observed patterns to infer changes in the frailty structure and biological (as distinct from chronological) age of the population. Our results reveal distinct patterns in both relative and absolute post-capture longevity of $C x$. pipiens

\section{REFERENCES}

Alto, B. W., Richards, S. L., Anderson, S. L., and Lord, C. C. (2014). Survival of West Nile virus-challenged Southern house mosquitoes, Culex pipiens quinquefasciatus, in relation to environmental temperatures. J. Vector Ecol. 39, 123-133. doi: 10.1111/j.1948-7134.2014.12078.x that heretofore have never been documented for any mosquito species. More generally, our paper introduces to the medical entomology literature the concept that useful demographic information can be derived, not just from traditional methods involving the analysis of dead mosquitoes, but from individuals that are captured live from the wild and maintained in the laboratory through their death.

Our study has at least two limitations. One limitation is that the study covered only a single field season. Thus, similar follow-up investigations will be needed to determine whether the trends in post-capture longevity that we observed are general or idiosyncratic. A second limitation concerns the confounding of frailty determinants. Population aging is clearly a universal source of frailty (and thus reduced post-capture longevity) in all populations. However, for mosquitoes, larval cycles will shape the later fitness of adults, host abundance can impact adult longevity through the availability of blood meals, and chance events such as heat spikes or freezing spells can selective kill-off different life stages. Although the impact each of these has on the remaining longevity of captured mosquitoes cannot be disaggregated in this study and may even be impossible in future studies, data on the observed changes in post-capture longevity in mosquito populations create new opportunities for hypothesis testing that have heretofore not been available.

\section{AUTHOR CONTRIBUTIONS}

$\mathrm{NP}, \mathrm{CI}$, and JC conceived and designed the experiments; NP and CI performed the experiments; HJ, HM, and JW analyzed the data, and NP, JC, SL, and EL interpreted data, constructed table and graphics, and wrote the paper.

\section{FUNDING}

Research part of Surveillance and Control Programme for West Nile Virus and malaria in Greece funded by National Strategic Reference Framework (NSRF) 2007-2013, co-funded by Greece and the European Union-European Regional Development (NP). Additional funding sources includes NIH P01 AG0876110 (JC), 1R56AG043995 (JC), NSF DMS-1104426 (HM) and NSF DMS-1407852 (JW).

\section{ACKNOWLEDGMENTS}

We thank William Reisen for his editorial comments and particularly for his input on aging methods in mosquitoes, and Demosthenes Daenas (N. Angchialos Volos) for technical support. 
Brownstein, J. S., Hett, E., and O'Neill, S. L. (2003). The potential of virulent Wolbachia to modulate disease transmission by insects. J. Invertebr. Pathol. 84, 24-29. doi: 10.1016/S0022-2011(03)00082-X

Caraballo, H. and King K. (2014). Emergency department management of mosquito-borne illness: Malaria, dengue, and West Nile virus. Emerg. Med. Pract. 16, 1-23.

Carey, J. R., Müller, H.-G., Wang, J.-L., Papadopoulos, N. T., Diamantidis, A., and Kouloussis, N. A. (2012a). Graphical and demographic synopsis of the captive cohort method for estimating population age structure in the wild. Exp. Gerontol. 47, 787-791. doi: 10.1016/j.exger.2012. 06.012

Carey, J. R., Papadopoulos, N. T., Papanastasiou, S., Diamanditis, A., and Nakas, C. T. (2012b). Estimating changes in mean population age using the death distributions of live-captured medflies. Ecol. Entomol. 37, 359-369. doi: 10.1111/j.1365-2311.2012.01372.x

Cook, P. E., Hugo, L. E., Iturbe-Ormaetxe, I., Williams, C. R., Chenoweth, S. F., Ritchie, S. A., et al. (2006). The use of transcriptional profiles to predict adult mosquito age under field conditions. Proc. Natl. Acad. Sci. 103, 18060-18065. doi: $10.1073 /$ pnas.0604875103

Cook, P. E., McMeniman, C. J., and O'Neil, S. L. (2008). Modifying insect population age structure to control vector-borne disease. Adv. Exp. Med. Biol. 627, 126-140. doi: 10.1007/978-0-387-78225-6_11

Dao, A., Yaro, A. S., Diallo, M., Timbiné, S., Huestis, D. L., Kassogué, Y., et al. (2014). Signatures of aestivation and migration in Sahelian malaria mosquito populations. Nature 516, 387-390. doi: 10.1038/nature13987

Denlinger, D. L., and Armbruster, P. A. (2014). Mosquito Diapause. Annu. Rev. Entomol. 59, 73-93. doi: 10.1146/annurev-ento-011613-162023

Desena, M. L., Edman, J. D., Clark, J. M., Symington, S. B., and Scott, T. W. (1999). Potential for aging female Aedes aegypti (Diptera: Culicidae) by gas chromatographic analysis of cuticular hydrocarbons, including a field evaluation. J. Med. Entomol. 36, 824-830. doi: 10.1093/jmedent/36.6.824

Fox, A. S., and Brust, R. A. (1994). How do dilatations for in mosquito ovarioles? Parasitol. Today 10, 19-23.

Frajollahi, A., Fonseca, D. M., Kramer, L. D., and Kilpatrick, A. M. (2011). Bird biting mosquitoes and human diseases: a review of the role of Culex pipiens complex mosquitoes in epidemiology. Infect. Genet. Evol. 11, 1577-1585. doi: 10.1016/j.meegid.2011.08.013

Gomes, B., Kioulos, E., Papa, A., Almeida, A. P., Vontas, J., and Pinto, J. (2013). Distribution and hybridization of Culex pipiens forms in Greece during the West Nile virus outbreak of 2010. Infect. Genet. Evol. 16, 218-225. doi: 10.1016/j.meegid.2013.02.006

Gomez, C., Rabinovich, J. E., and Machado-Allison, C. E. (1977). Population analysis of Culex pipiens fatigans Wid. (Diptera: Culicidae) under laboratory conditions. J. Med. Entomol. 13, 453-463. doi: 10.1093/jmedent/13.4-5.453

James, S., Takken, W., Collins, F. H., and Gottlieb, M. (2013). Perspective piece: needs for monitoring mosquito transmission of malaria in a pre-elimination world. Am. J. Trop. Med. Hyg. doi: 10.4269/ajtmh.13-0175

Joy, T. K., Gutierrez, E. H. J., Ernst, K., Walker, K. R., Carriere, Y., Torabi, M., et al. (2012). Aging field collected Aedes aegypti to determine their capacity for dengue transmission in the southwestern United States. PLoS ONE 7:e46946. doi: 10.1371/journal.pone.0046946

Klowden, M. J., and Lea, A. O. (1980). "Physiologically old" mosquitoes are not necessarily old physiologically. Am. J. Trop. Med. Hyg. 29, 1460-1464.

Kouloussis, N. A., Papadopoulos, N. T., Müller, H.-G., Wang, J.-L., Mao, M., Katsoyannos, B. I., et al. (2009). Life table assay for assessing relative age bias in medfly capture methods. Entomol. Exp. Appl. 132, 172-181. doi: 10.1111/j.1570-7458.2009.00879.x

Lardeux, F., Ung, A., and Chebret, M. (2000). Spectrofluorometers are not adequate for aging Aedes and Culex (Diptera: Culicidae) using pteridine fluorescence. J. Med. Entomol. 37, 769-773. doi: 10.1603/0022-2585-37.5.769

Levine, M. E. (2012). Modeling the rate of senescence: can estimated biological age predict mortality more accurately than chronological age? J. Gerontol. Biol. Sci. 68, 667-674. doi: 10.1093/gerona/gls233

MacDonald, G. (1957). The Epidemiology and Control of Malaria. Oxford: Oxford University Press.

Mayagaya, V. S., Michel, K., Benedict, M. Q., Killeen, G. F., Wirtz, R. A., Ferguson, H. M., et al. (2009). Non-destructive determination of age and species of
Anopheles gambiae s.l. using near-infrared Spectroscopy. Am. J. Trop. Med. Hyg. 81, 622-630. doi: 10.4269/ajtmh.2009.09-0192

McMeniman, C. J., Lane, R. V., Cass, B. N., Fong, A. W. C., Sidhu, M., Wang, Y.-F., et al. (2009). Stable introduction of a life-shortening Wolbachia infection into the mosquito Adedes aegypti. Science 323, 141-144. doi: 10.1126/science.1165326

Mia, M. S., Begum, R. A., Er, A. C., Abidin, R. D. Z. R. Z., and Pereira, J. J. (2012). Burden of malaria at household level: a baseline review in the advent of climate change. J. Environ. Sci. Technol. 5, 1-15. doi: 10.3923/jest.2012.1.15

Müller, H. G., Wang, J.-L., Carey, J. R., Caswell-Chen, E. P., Chen, C., Papadopoulos, N., et al. (2004). Demographic window to aging in the wild: constructing life tables and estimating survival functions from marked individuals of unknown age. Aging Cell 3, 125-131. doi: 10.1111/j.14749728.2004.00096.x

Nasci, R. S., Savage, H. M., White, D. J., Miller, J. R., Cropp, B. C., Godsey, M. S., et al. (2001). West Nile virus in overwintering Culex mosquitoes, New York City, 2000. Emerging Infect. Dis. 7, 742-744. doi: 10.3201/eid0704.0 17426

Nelson, R. L., and Milby, M. M. (1982). Autogeny and blood-feeding by Culex tarsalis (Diptera:Culicidae) and the interval between oviposition and feeding. Can Entomol. 114, 515-521. doi: 10.4039/Ent114515-6

Pervanidou, D., Detsis, M., Danis, K., Mellou, K., Papanikolaou, E., Terzaki, I., et al. (2014). West Nile virus outbreak in humans, Greece, 2012: Third consecutive year of local transmission. Euro. Surveill. 19, pii=20758. doi: 10.2807/15607917.ES2014.19.13.20758

Reisen, W. K., Mahmood, F., and Parveen, T. (1980). Anopheles culicifacies Giles: a release-recapture experiment with cohorts of known age with implications for malaria epidemiology and genetical control in Pakistan. Trans. R. Soc. Trop. Med. Hyg. 74, 307-317. doi: 10.1016/0035-9203(80)90089-9

Reisen, W. K., Mahmood, F., and Azra, K. (1981). Anopheles culicifacies Giles: adult ecological parameters measured in rural Punjab Province, Pakistan using capture-mark-release-recapture and dissection methods, with comparative observations on An. stephensi Liston and An. subpictus Grassi. Res. Popul. Ecol. 23, 39-60. doi: 10.1007/BF02514092

Styer, L. M., Carey, J. R., Wang, J.-L., and Scott, T. W. (2007). Mosquitoes do senesce: departure from the paradigm of constant mortality. Am. J. Trop. Med. Hyg. 76, 111-117.

Tauber, M. J., Tauber, C. A., and Masaki, S. (1986). Seasonal Adaptations of Insects. New York, NY: Oxford University Press.

Vinogradova, E. B. (2000). Culex pipiens pipiens Mosquitoes: Taxonomy, Distribution, Ecology, Physiology, Genetics, Applied Importance and Control. Sofia: Pensoft Publishers.

Vogel, G. (2016). Evidence grows for Zika virus as pregnancy danger. Science 351, 1123-1124. doi: 10.1126/science.351.6278.1123

Walter, W. M., and Hacker, C. S. (1974). Variation in life table characteristics among three geographic strains of Culex pipiens quinquefasciatus. J. Med. Entomol. 11, 541-550.

Wang, J. (1998). Analysis of life table of organophosphate-resistant strains of Culex pipiens pallens. Entomol. Sin. 5, 159-165. doi: 10.1111/j.17447917.1998.tb00315.x

Wang, M.-H., Marinotti, O., Zhong, D., James, A. A., Walker, E., Guda, T., et al. (2013). Gene expression-based biomarkers for Anopheles gambiae age grading. PLoS ONE 8:e69439. doi: 10.1371/journal.pone.0069439

WHO (2014). A Global Brief on Vector-Borne Diseases. WHO Reference Number: DC/WHD/2014. Geneva: World Health Organization.

Conflict of Interest Statement: The authors declare that the research was conducted in the absence of any commercial or financial relationships that could be construed as a potential conflict of interest.

Copyright (C) 2016 Papadopoulos, Carey, Ioannou, Ji, Müller, Wang, Luckhart and Lewis. This is an open-access article distributed under the terms of the Creative Commons Attribution License (CC BY). The use, distribution or reproduction in other forums is permitted, provided the original author(s) or licensor are credited and that the original publication in this journal is cited, in accordance with accepted academic practice. No use, distribution or reproduction is permitted which does not comply with these terms. 